\title{
Relação bidirecional entre doença periodontal e o diabetes mellitus - revisão de literatura
}

\author{
Bi-directional relationship between periodontal disease and diabetes mellitus - a literature \\ review
}

Relaciónbidireccional entre periodontitis y diabetes mellitus - revisión de la literatura

Maria Caroline Rios Piecha ${ }^{1}$, Caroline Fernandes e Silva ${ }^{1}$, Taciane Menezes da Silveira ${ }^{1}$, Natália Marcumini Pola ${ }^{1 *}$.

\section{RESUMO}

Objetivo: Revisar a literatura abrangendo a importância da inter-relação entre a doença periodontal e o diabetes melittus. Resultados: Os resultados demonstraram que a relação bidirecional entre o diabetes mellitus e a doença periodontal se mostra de alta prevalência na população. Fatores etiológicos e mecanismos fisiológicos comprovam a interferência de uma doença na progressão da outra. Os resultados demonstram ainda que a terapia periodontal não cirúrgica pode auxiliar no controle glicêmico com uma redução de 0,2 a $0,4 \%$ na HbA1c. Para o sucesso deste tratamento em pacientes diabéticos, é preciso aliar o controle da condição sistêmica, com o uso correto de medicamentos prescritos e estilo de vida saudável. Conclusão: Inúmeros estudos descritos relatam a importância do acompanhamento odontológico em pacientes diabéticos, tanto pela manutenção da saúde bucal, quanto na colaboração do controle glicêmico. Cabe destacar também a relevância da necessidade de uma abordagem multidisciplinar dos profissionais da saúde, para o controle dessas condições que atingem elevada parcela da população, assim como o esclarecimento dos pacientes do benefício do tratamento para ambas as condições.

Palavras-chave: Doença periodontal, Diabetes mellitus, Glicemia.

\begin{abstract}
Objective: To review the literature reporting the importance of the relationship between periodontal disease and diabetes mellitus. Results: The results showed that the bidirectional relationship between diabetes mellitus and periodontal disease is highly prevalent in the population. Etiological factors and physiological mechanisms prove the interference of one disease in the progression to another. The results also showed that non-surgical periodontal therapy can assist in glycemic control with a 0.2 to $0.4 \%$ reduction in $\mathrm{HbA} 1 \mathrm{c}$. For the success of this treatment in diabetic patients, it is necessary to combine the systemic condition control with the correct use of medical drugs and a healthy lifestyle. Conclusion: Numerous studies described the importance of dental follow-up in diabetic patients, both for the maintenance of oral health and for the collaboration of glycemic control. It is also worth highlighting the relevance of the need for a multidisciplinary approach of health professionals to control these conditions that affect a large portion of the population, as well as to clarify patients aboutthe benefit of treatment for both conditions.
\end{abstract}

Keywords: Periodontal disease, Diabetes mellitus, Blood glucose.

\section{RESUMEN}

Objetivo: Revisar la literatura que cubre la importancia de la interrelación entre la enfermedad periodontal y la diabetes melittus. Resultados: Los resultados mostraron que la relación bidireccional entre diabetes mellitus y enfermedad periodontal muestra una alta prevalencia en la población. Los factores etiológicos y

\footnotetext{
${ }^{1}$ Faculdade de Odontologia (UFPel), Pelotas - RS. *E-mail: nataliampola@gmail.com
} 
los mecanismos fisiológicos prueban la interferencia de una enfermedad en la progresión de otra. Los resultados también demuestran que la terapia periodontal no quirúrgica puede ayudar en el control glucémico con una reducción de 0.2 a $0.4 \%$ en $\mathrm{HbA} 1 \mathrm{c}$. Para el éxito de este tratamiento en pacientes diabéticos, es necesario combinar el control de la condición sistémica con el uso correcto de medicamentos recetados y un estilo de vida saludable. Conclusión: Numerosos estudios describieron la importancia del seguimiento dental en pacientes diabéticos, tanto para el mantenimiento de la salud oral como para la colaboración del control glucémico. También vale la pena resaltar la relevancia de la necesidad de un enfoque multidisciplinario de los profesionales de la salud para controlar estas afecciones que afectan a una gran parte de la población, así como para aclarar a los pacientes sobre el beneficio del tratamiento para ambas afecciones.

Palabras clave: Enfermedad periodontal, Diabetes mellitus, Glucosa en sangre.

\section{INTRODUÇÃO}

O Diabetes Mellitus (DM) é uma doença crônica metabólica, gerada pela falta de secreção do hormônio insulina ou pela falha na ação deste hormônio. Produzida pelo pâncreas, a insulina é responsável pela redução da glicemia, ao promover a entrada de glicose nas células (ADA, 2019). Quando há falha de sua função, os níveis de glicose sanguínea aumentam, causando o estado de hiperglicemia crônica, principal característica do diabetes.

Esta condição apresenta dois tipos considerados os principais: diabetes mellitus tipo 1 (DM1), diabetes mellitus tipo 2 (DM2). O DM1 é caracterizado por uma resposta autoimune do organismo que leva a produção deficiente ou inexistente de insulina, sendo necessária a reposição deste hormônio diariamente. Sua causa está associada a fatores genéticos, sendo os pacientes jovens a população mais afetada (ROGLIC G, 2016).

No DM2 ocorre a falha na ação da insulina pelo aumento exacerbado da produção deste hormônio, com o intuito de reverter a hiperglicemia instalada. Assim, desencadeia a resistência do próprio organismo à insulina. Segundo a Associação Americana de Diabetes, o DM2 é o mais comum, prevalecendo em $90 \%$ dos casos (ADA, 2019). Por apresentar-se assintomático, geralmente quando diagnosticado o paciente já apresenta outras complicações associadas ao quadro hiperglicêmico. Condições de sobrepeso, sedentarismo, idade avançada e características genéticas são os principais fatores de risco para ocorrência de DM2 (ADA, 2019).

Dados da Federação Internacional de Diabetes mostram que em 2017 houve 425 milhões de pessoas diabéticas no mundo e a estimativa para 2045 seria de um aumento de 48\% (IDF, 2017). No Brasil, 6,2\% da população referiu diagnóstico de diabetes, o equivalente a 9,1 milhões de pessoas (IBGE, 2014).

O estado de hiperglicemia crônica pode gerar alterações no sistema imunológico do indivíduo, aumentando as citocinas pró-inflamatórias, e resultando em um indivíduo mais suscetível a doenças oportunistas (NASCIMENTO GG, et al., 2018). Nesse contexto, as principais complicações associadas ao DM são as doenças cardiovasculares, retinopatia, insuficiência renal, neuropatia e alterações bucais (ROGLIC G, 2016), sendo o principal exemplo desta última a Doença Periodontal (DP), que é considerada a sexta maior complicação do DM (LOE H, 1993).

A DP se desenvolve por meio de um processo inflamatório específico, induzido por patógenos periodontais e acomete inicialmente o tecido gengival. $\mathrm{O}$ acúmulo de biofilme a longo prazo resulta em sua maturação, gerando um quadro de inflamação persistente com perda irreversível das estruturas de suporte dos dentes, caracterizando a periodontite (CATON JG, et al., 2018).

A DP atinge atualmente $7,4 \%$ da população mundial, sendo considerada a sexta doença mais prevalente no mundo (KASSEBAUM NJ, et al., 2017). Na etiopatogenia da doença, torna-se importante relatar que a DP pode ser influenciada por uma série de fatores de risco, incluindo condições sistêmicas como diabetes, imunodeficiência, estresse, hipertensão e osteoporose (NASCIMENTO GG, et al., 2018). 
Estudos longitudinais têm demonstrado que a relação DM e DP é bidirecional, sendo observado um agravo na destruição do tecido periodontal em indivíduos diabéticos e um pior controle glicêmico em pacientes diabéticos com DP (COSTA KL, et al., 2017; TESHOME A e YITAYEH A, 2016). A melhora no controle glicêmico após o tratamento periodontal foi observada principalmente em pacientes com DM2 (TSOBGNY-TSAGUE NF, et al., 2018; AHUJA CR, et al., 2019). Estudos in vitro demonstraram um efeito direto da hipo/hiperglicemia nas células periodontais e o efeito adverso indireto da hiperglicemia estimulando as células do sistema imunológico na liberação de citocinas inflamatórias, colaborando para o desenvolvimento ou agravamento da DP (NISHIMURA F, et al., 1998). Os estudos realizados para avaliar o efeito da terapia periodontal na melhora dos índices glicêmicos ainda são recentes e contraditórios. Vergnes $\mathrm{JN}$ et al. (2018) e Almeida ML et al. (2020) não relataram efeitos do tratamento periodontal nos níveis de hemoglobina glicada (HbA1c) em pacientes com DM2.

Contraditoriamente, autoresapoiam a eficácia da terapia periodontal e relatam haver uma redução de HbA1c de aproximadamente $0,4 \%$, após a abordagem periodontal não cirúrgica (AHUJA CR, et al., 2019). Todavia, ensaios clínicos controlados e randomizados, bem como estudos longitudinais, são necessários para esclarecer essas evidências. Diante do contexto apresentado, este trabalho tem como objetivo abordar a importância da inter-relação DM e DP, destacando seus possíveis mecanismos de associação por meio de uma revisão de literatura.

\section{REVISÃO BIBLIOGRÁFICA}

\section{Relação bidirecional entre DM e DP}

A DM e DP apresentam uma relação altamente complexa caracterizada como bidirecional (NASCIMENTO GG, et al., 2018) que tem sido extensivamente explorada nas últimas décadas em diversos estudos epidemiológicos e experimentais. A DM quando presente conduz a um risco elevado de desenvolvimento e/ou progressão da periodontite. Indivíduos diabéticos tendem a desenvolver resposta ao acúmulo bacteriano na gengiva de forma precoce e hiperinflamatória, quando comparados a não diabéticos, desencadeando maior perda de inserção clínica (LALLA E e PAPAPANOU P, 2011).

Diversos estudos avaliando esta relação entre as condições foram realizados em uma comunidade de Índios Pima no estado do Arizona, EUA. Um levantamento feito em 2.273 indivíduos dessa comunidade encontrou 2,6 vezes maior ocorrência de DP em portadores de DM do que entre os não diabéticos (NELSON RG, et al., 1990). Além disso, foi visto que em relação ao DM2, houve maior predominância de DP avançada, e que a perda de inserção periodontal e a perda óssea alveolar ocorriam de forma precoce e em intensidade maior do que em indivíduos saudáveis (LOE H, 1993).

O controle da glicemia em indivíduos diabéticos é medido por meio dos níveis de hemoglobina glicada (HbA1c), no intervalo de 3 meses. Estes níveis devem ser inferiores a 5,7\% em indivíduos não diabéticos, com variações individuais (ADA, 2019). Entretanto, o descontrole glicêmico parece ser um importante fator que irá influenciar o início e a progressão da DP (ADA, 2019). Um acompanhamento prospectivo na mesma população de Índios Pima demonstrou associação entre o controle glicêmico inadequado e a prevalência significativa de perda óssea alveolar progressiva, quando comparado a um grupo com controle glicêmico adequado (TAYLOR GW, et al., 1996). O controle glicêmico inadequado foi reportado ser responsável pelo aumento de 2,9 vezes o desenvolvimento de periodontite em indivíduos adultos com DM2e ao aumento de 86\% no risco de progressão da periodontite (ADA, 2019; NASCIMENTO GG, et al., 2018).

Diante dessas evidências, é possível compreender a inter-relação do DM com o início e progressão da periodontite através de aspectos fisiológicos. No estado hiperglicêmico crônico, há o aumento dos níveis de leucócitos e marcadores pró-inflamatórios imunológicos, desencadeando uma inflamação sistêmica crônica. Esta condição pode modificar de forma deletéria a fisiologia do periodonto, levando a exarcebação do quadro inflamatório destes tecidos (TAYLOR JJ, et al., 2013). No entanto, devido ao perfil infectoinflamatório da DP, os índices glicêmicos de pacientes diabéticos também podem ser comprometidos quando em presença da DP, pelo aumento da produção de mediadores pró-inflamatórios como TNF-a, IL6, IL1 e de proteínas de fase-aguda, que prejudicam a sinalização intracelular da insulina (LLAMBÉS F, et al., 2015). 
Alguns estudos de coorte demonstraram que a periodontite foi consideravelmente associada a um controle glicêmico insatisfatório, medido pela $\mathrm{HbA1c}$. Este risco foi mais acentuado nos pacientes que apresentavam pior índice de $\mathrm{HbA1c}$ no início do estudo. Além disso, maior resistência à insulina foi reportada em pessoas com periodontite (GRAZIANI F, et al., 2018). Costa KL et al. (2017) avaliou periodontalmente 80 pacientes com DM2 em dois momentos, com intervalo médio de 38 meses. Foi observado que o aumento da $\mathrm{HbA1c}$ ao longo do tempo foi significativo quando o quadro de periodontite severa foi diagnosticado no início do estudo (COSTA KL, et al., 2017).

Ainda, um estudo prospectivo com 628 indivíduos diabéticos na população de índios Pima com um acompanhamento médio de 11 anos, demonstrou um risco consideravelmente maior para mortalidade cardíaca e renal nestes indivíduos quando eles apresentavam o quadro de periodontite associado (SAREMI A, et al., 2005). Atualmente tem se estabelecido a hipótese de que a DP poderia, ao elevar os níveis glicêmicos, gerar um quadro de pré-diabetes em pacientes não diabéticos (POLAK D e SHAPIRA L, 2018). Um estudo longitudinal reportou que pacientes não diabéticos com DP apresentaram níveis glicêmicos elevados após 5 anos. Os autores atribuíram esse aumento ao status periodontal, pois nos pacientes que apresentavam maior número de sítios periodontais com perda de inserção maior ou igual a $5 \mathrm{~mm}$, o aumento da glicemia foi expressivamente maior, sendo a severidade da DP considerada um prenunciador da progressão de HbA1c (DEMMER RT, et al., 2010).

\section{Mecanismos fisiopatológicos associados ao DM na DP}

Para entender como funciona a inter-relação entre essas duas doenças é necessário compreender a biologia envolvida neste processo e identificar os fatores associados a bidirecionalidade entre DM e DP. Muitos dos mecanismos pelos quais o DM influencia o periodonto têm fisiopatologia similar à das clássicas complicações micro e macrovasculares observadas com frequência nesses pacientes (ENGEBRETSON $S$ e KOCHER T, 2013). As citocinas inflamatórias (IL-1, IL-6, TNF- $\alpha$ ), produção de Proteína C Reativa (PCR), estresse oxidativo e o ligante do receptor ativador do fator nuclear kappa-B (RANKL) estão elevadas em indivíduos com DM e periodontite, em comparação com aqueles que apresentam apenas periodontite (ADA, 2019).

Como característica do DM, o estado inflamatório induz a resistência à insulina, aumentando assim as concentrações dos marcadores inflamatórios TNF- $\alpha$ e IL-6, produzidos por monócitos e macrófagos no tecido adiposo (TAYLOR JJ, et al., 2013). Essas citocinas possuem vários efeitos sobre a sensibilidade à insulina, assim como estimulam a produção de PCR, que está relacionada a maiores ocorrências de doenças cardiovasculares e resistência à insulina. Assim, essas alterações nos níveis insulínicos desencadeiam um quadro hiperglicêmico, que gera maior destruição nos tecidos periodontais (LLAMBÉS F, et al., 2015).

Alterações na hemostasia do osso alveolar e a formação de produtos finais de glicosilação (AGEs) são resultantes da hiperglicemia associada ao DM. Os AGEs desempenham um papel pró-inflamatório e oxidativo nas células (COSTA KL, et al., 2017). Por conseguinte, esses fatores inflamatórios em comum produzem um efeito somatório quando ambas as doenças estão presentes, o que dificulta 0 controle glicêmico e a melhora clínica da DP.

Um dos meios de regulação do metabolismo ósseo é o sistema RANKL/OPG. O RANKL é um marcador de reabsorção óssea, e a sua produção funciona em resposta a presença de citocinas inflamatórias (POLAK D e SHAPIRA L, 2018). A ligação de RANKL com o seu receptor leva a formação de osteoclastos, ocasionando maior reabsorção óssea. A osteoprotegerina (OPG) produzida por osteoblastos, pode ligar-se à RANKL para inibir a ativação de osteoclastos (POLAK D e SHAPIRA L, 2018). No entanto, em indivíduos diabéticos, todo este processo encontra-se alterado, podendo agravar o quadro periodontal.

\section{Citocinas inflamatórias e função celular imune}

Fatores inflamatórios, resposta do hospedeiro alterada e a resistência à insulina parecem ser os principais aspectos influenciadores na patogênese do DM2 e suas implicações (TESHOME A e YITAYEH A, 2016). As citocinas se fazem importantes na resposta imune e inflamatória de diabéticos, podendo 
determinar o nível de infecção periodontal. Alterações sistêmicas inflamatórias são relevantes para a patogênese do DM2, que podem influenciar a deficiência insulínica e, portanto, levar a progressão do estado diabético (LONGO PL, et al., 2018).

Alguns estudos relatam níveis alterados de citocinas no fluido crevicular gengival (FCG), saliva e tecido gengival de pacientes diabéticos tipo 2 com periodontite crônica, em comparação com pacientes sistemicamente saudáveis. Os dados mais relevantes estão relacionados à níveis aumentados de TNF- $\alpha$, IL-6 e IL-1 (HUANG X, et al., 2015). Estudos experimentais em animais têm destacado a importância do TNF- $\alpha$ em possivelmente prolongar a resposta imune à ação da placa bacteriana no quadro de DM2, o que pode contribuir com o desenvolvimento e progressão da periodontite (PACIOS S, et al., 2012). A redução dos níveis de algumas citocinas por inibição específica do TNF- $\alpha$ levantou a hipótese de que a desregulação da associação entre esses dois fatores pode representar um mecanismo pelo qual o DM2 poderia alterar a resposta do hospedeiro ao desafio bacteriano.

Com relação a função imunológica, como já foi descrito, neutrófilos depois de realizarem suas funções de proteção são hipofuncionais, porém quando estão alterados junto a monócitos e macrófagos hiperresponsivos, podem ser responsáveis pela destruição periodontal gerada (TAYLOR JJ, et al., 2013). Mediadores pró-inflamatórios como citocinas e metaloproteínases de matriz (MMPs), podem estar associados ao nível de controle glicêmico em diabéticos. É a resposta do hospedeiro ao desafio bacteriano que desencadeia a maior gravidade da DP em pacientes diabéticos (NASCIMENTO GG, et al., 2018).

\section{Hiperglicemia relacionada ao AGE/RAGE e Homeostase do osso alveolar}

DM2 e suas complicações estão associados a hiperglicemia e sua produção de AGEs, e essas mudanças levam a desregulação imune manifestada por um estado inflamatório crônico (COSTA KL, et al., 2017). A hiperglicemia induz a produção de AGEs, que podem se ligar a receptores específicos RAGE nos macrófagos, fibroblastos e células endoteliais. A partir deste processo, os macrófagos são transformados em células hiper-reativas que produzem citocinas, como as IL-1 e IL-6, TNF- $\alpha$ e estresse oxidativo(GÓRSKA R, et al., 2003).Os AGEs alteram células endoteliais que se tornarão hiperpermeáveis e hiperexpressivas, enquanto os fibroblastos terão prejuízos na produção do colágeno. Portanto, os AGEs produzidos pela hiperglicemia crônica podem produzir respostas hiperinflamatórias, modificações vasculares, cicatrização alterada e aumento da predisposição a infecções (LLAMBÉS F, et al., 2015). Ao mesmo tempo, o DM inadequadamente controlado é conhecido pela alta formação de estresse oxidativo, que causa necrose celular ou destruição em tecidos ósseos.Sabe-se que este estado de estresse estimula diretamente as vias associadas à produção de citocinas pró-inflamatórias, resistência à insulina e complicações do diabetes (ALLEN EM e CHAPPLE IL, 2012).

A expressão dos AGEs, juntamente com marcadores do estresse oxidativo foi demonstrada em tecidos gengivais de pacientes diabéticos com periodontite (SCHMIDT AM, et al., 1996). Posteriormente, os AGEs foram encontrados na saliva de pacientes diabéticos e associados aos níveis de placa bacteriana (YOON M, et al., 2004). Além disso, os níveis plasmáticos de AGEs mostraram-se significativamente associados à extensão da periodontite em indivíduos com DM2. Em humanos, os AGEs têm demonstrado significativa expressividade no periodonto de pacientes diabéticos e tem sido relacionado $\mathrm{com}$ a deterioração periodontal (ZIZZI A, et al., 2013).

Com relação aos marcadores ósseos OPG e RANKL, é importante destacar que também estão associados ao controle glicêmico descontrolado no DM2 em pacientes com periodontite (TAYLOR JJ, et al., 2013). Estudos clínicos demonstraram que a expressão de RANKL/OPG são maiores em pacientes diabéticos mal controlados com periodontite em comparação com indivíduos bem controlados (RIBEIRO FV, et al., 2011; WU Y, et al., 2015). Em síntese, evidências de estudos de mediadores de reabsorção óssea RANKL/OPG sugerem fortemente que a homeostase óssea alveolar alterada é uma via importante na patogênese da periodontite no diabetes (POLAK D e SHAPIRA L, 2018).

\section{Periodontite influenciando no quadro diabético}

A periodontite tem sido associada com pior controle glicêmico em pessoas com diabetes (GRAZIANI F, et al., 2018), aumentando o risco de surgirem complicações diabéticas. Na presença de periodontite, 
observa-se a elevação da produção de mediadores pró-inflamatórios como TNF-a, IL-6, IL-1, que são antagonistas da insulina. Também se eleva o estresse oxidativo e PCR, os quais quando alterados podem levar a resistência à insulina (SANZ M, et al., 2018). Alterações na HbA1c não são induzidas diretamente pela inflamação, mas sim através da hiperglicemia causada pela resistência à insulina. Por conseguinte, esta pode ser uma das vias de associação do quadro periodontal às altas taxas de HbA1c (LLAMBÉS F, et al., 2015). Além disso, infecções periodontais podem promover aumento dos níveis de citocinas próinflamatórias no DM tanto local como sistemicamente (POLAK D e SHAPIRA L, 2018).

Um estudo longitudinal que avaliou pacientes com periodontite por 5 anos demonstrou um aumento nos níveis de HbA1c, com resultados maiores em indivíduos com níveis elevados de PCR, o que pode apontar a relação entre a periodontite e a inflamação sistêmica (DEMMER RT, et al., 2010). Marcadores do estresse oxidativo sistêmico tem se mostrado elevados tanto no diabetes quanto na periodontite, e estão associados com a expressão de PCR nos pacientes com periodontite quando comparados a controles saudáveis (D'AIUTO F, et al., 2010).

\section{Importância do tratamento periodontal no controle glicêmico}

O efeito das infecções periodontais no DM é teoricamente elucidado pelo aumento de mediadores próinflamatórios sistêmicos, que estão envolvidos em mecanismos que resultam na resistência da insulina, exacerbando o quadro hiperglicêmico já existente no diabético (LALLA E e PAPAPANOU P, 2011). Assim, na ausência de controle glicêmico eficiente, há a possiblidade de instalação das complicações relacionadas ao diabetes (D'AIUTO FD, et al., 2017).

Ao avaliar o efeito do tratamento periodontal convencional em indivíduos diabéticos, uma revisão sistemática recente reportou um impacto da raspagem e alisamento radicular na redução significativa dos níveis de PCR em pacientes com DM2 (BAEZA M, et al., 2020). Embora poucos estudos que avaliem este parâmetro tenham sido desenvolvidos, os autores apontam que estes resultados são consistentes entre si e com outros trabalhos na literatura. A terapia periodontal não cirúrgica tem sido correlacionada ao melhor balanço do estresse oxidativo e melhoras significativas na qualidade de vida dos indivíduos diabéticos (MIZUNO H, et al., 2017). No entanto, a diminuição dos níveis de hemoglobina glicada não é um resultado observado em muitos estudos clínicos, apesar de ser apontado que o tratamento periodontal possibilita uma diminuição dos níveis de inflamação circulatória, o que pode auxiliar no controle glicêmico (MIZUNO H, et al., 2017).

Dessa forma, a terapia periodontal não cirúrgica de raspagem e alisamento radicular poderia influenciar na melhora hiperglicêmica a partir da redução das citocinas presentes na inflamação periodontal, podendo estimular uma melhora na sensibilidade à insulina, e assim contribuir tanto com o quadro periodontal quanto com o diabetes e suas implicações (TESHOME A, et al., 2016). Estima-se que uma redução média de 0,20\% nos níveis de HbA1c está associada a uma redução de $10 \%$ na mortalidade dos indivíduos (KHAW $\mathrm{KT}$, et al., 2004).

\section{CONSIDERAÇÕES FINAIS}

A relação bidirecional entre o diabetes mellitus e a doença periodontal se mostra de suma relevâncianos cenários médico e odontológico atuais. Ao longo deste trabalho pode-se compreender que os fatores etiológicos e mecanismos fisiológicos envolvidos nestas condições estão associados, fazendo com que uma doença interfira no desenvolvimento e progressão da outra. Inúmeros estudos descritos apontam para a importância do acompanhamento odontológico de pacientes diabéticos, tanto pelo aspecto de saúde bucal, quanto para colaborar junto a outros fatores para um melhor controle glicêmico e qualidade de vida destes pacientes.

\section{REFERÊNCIAS}

1. ADA. American Diabetes Association. 2. Classification and Diagnosis of Diabetes: Standards of Medical Care in Diabetes-2019. Diabetes Care. 2019; 42(Suppl 1):S13-S28. 
2. AHUJA CR, et al. Effect of non-surgical periodontal treatment on gingival crevicular fluid and serum leptin levels in periodontally healthy chronic periodontitis and chronic periodontitis patients with type 2 diabetes mellitus. J InvestigClin Dent. 2019; 10(3):e12420.

3. ALLEN EM, CHAPPLE IL. The Relationship Between Periodontitis and Glycaemic Control in Type 2 Diabetes. EurEndocrinol. 2012; 8(2):89-93.

4. ALMEIDA ML, et al. Effects of a full-mouth disinfection protocol on the treatment of type-2 diabetic and non-diabetic subjects with mild-to-moderate periodontitis: one-year clinical outcomes. Clin Oral Investig. 2020; 24(1):333-341.

5. ATLAS, IDF Diabetes. Eighth edition. Brussels, Belgium: International Diabetes Federation (IDF), p. $147,2017$.

6. BAEZA M, et al. Effect of periodontal treatment in patients with periodontitis and diabetes: systematic review and meta-analysis. J Appl Oral Sci. 2020; 28:e20190248.

7. CATON JG, et al. A new classification scheme for periodontal and peri-implant diseases, conditions-Introduction, and key changes from the 1999 classification. J ClinPeriodontol. 2018;45 Suppl20:S1-S8.

8. COSTA KL, et al. Influence of Periodontal Disease on Changes of Glycated Hemoglobin Levels in Patients With Type 2 Diabetes Mellitus: A Retrospective Cohort Study. J Periodontol. 2017; 88(1):17-25.

9. D'AIUTO FD, et al. Oxidative stress, systemic inflammation, and severe periodontitis. J Dental Res. 2010; 89(11):1241-1246.

10. D'AIUTO FD, et al. Evidence summary: The relationship between oral diseases and diabetes. Br Dent J. 2017; 222(12):944-948.

11. DEMMER RT, et al. Periodontal status and A1C change: longitudinal results from the study of health in Pomerania (SHIP). Diabetes Care. 2010; 33(5):1037-1043.

12. ENGEBRETSON S, KOCHER T. Evidence that periodontal treatment improves diabetes outcomes: a systematic review and meta-analysis. J ClinPeriodontol, 2013; 40(14):S153-S163.

13. GÓRSKA R, et al. Relationship between clinical parameters and cytokine profiles in inflamed gingival tissue and serum samples from patients with chronic periodontitis. J ClinPeriodontol, 2003; 30(12):1046-1052.

14. GRAZIANI F, et al. A systematic review and meta-analysis of epidemiologic observational evidence on the effect of periodontitis on diabetes An update of the EFP-AAP review. J ClinPeriodontol. 2018; 45(2):167-187.

15. HUANG X, et al. Hyperglucose contributes to periodontitis: involvement of the NLRP3 pathway by engaging the innate immunity of oral gingival epithelium. J Periodontol. 2015; 86(2):327-335.

16. IBGE. Instituto Brasileiro de Geografia e Estatística. Pesquisa Nacional de Saúde - PNS 2013: percepção do estado de saúde, estilos de vida e doenças crônicas. Rio de Janeiro. Ministério da saúde. 2014; 1:40-43.

17. KHAW KT, et al. Annals of Internal Medicine Article Association of Hemoglobin A1c with Cardiovascular Disease and Mortality in Adults: The European Prospective Investigation into Cancer in Norfolk. Ann Intern Med. 2004;141(12):413-420.

18. KASSEBAUM NJ, et al. Global, Regional, and National Prevalence, Incidence, and Disability-Adjusted Life Years for Oral Conditions for 195 Countries, 1990-2015: A Systematic Analysis for the Global Burden of Diseases, Injuries, and Risk Factors. J Dent Res. 2017; 96(4):380-387.

19. LALLA E, PAPAPANOU PN. Diabetes mellitus and periodontitis: A tale of two common interrelated diseases. Nat Rev Endocrinol. 2011; 7(12):738-748.

20. LLAMBÉS F, et al. Relationship between diabetes and periodontal infection. World J Diabetes. 2015; 6(7):927-935.

21. LOE H. Periodontal Disease: The sixth complication of diabetes mellitus. Diabetes Care. 1993; 16(1):329-334.

22. LONGO PL, et al. Glycaemic status affects the subgingival microbiome of diabetic patients. J ClinPeriodontol, 2018; 45(8): 932-940.

23. MIZUNO H, et al. The effects of non-surgical periodontal treatment on glycemic control, oxidative stress balance and quality of life in patients with type 2 diabetes: A randomized clinical trial. PLoS One. 2017; 12(11):e0188171.

24. NASCIMENTO GG, et al. Does diabetes increase the risk of periodontitis? A systematic review and meta-regression analysis of longitudinal prospective studies. ActaDiabetol. 2018; 55(7):653-667.

25. NELSON RG, et al. Periodontal disease and NIDDM in Pima Indians. Diabetes Care. 1990; 13(8):836-840.

26. NISHIMURA F, et al. Periodontal disease as a complication of diabetes mellitus. Ann Periodontol. 1998; 3(1):20-29.

27. PACIOS S, et al. Diabetes aggravates periodontitis by limiting repair through enhanced inflammation. FASEB J, 2012; 26:1423-1430.

28. POLAK D, SHAPIRA L. An update on the evidence for pathogenic mechanisms that may link periodontitis and diabetes. J ClinPeriodontol. 2018; 45(2):150-166.

29. RIBEIRO FV, et al. Cytokines and bone-related factors in systemically healthy patients with chronic periodontitis and patients with type 2 diabetes and chronic periodontitis. J Periodontol. 2011; 82(8):1187-1196.

30. ROGLIC G. WHO Global report on diabetes: A summary. Int J Non-Commun Dis. 2016;1:3-8.

31. SANZ M, et al. Scientific evidence on the links between periodontal diseases and diabetes: Consensus report and guidelines of the joint workshop on periodontal diseases and diabetes by the International Diabetes Federation and the European Federation of Periodontology. J ClinPeriodontol. 2018; 45(2):138-149.

32. SAREMI A, et al. Periodontal disease and mortality in type 2 diabetes. Diabetes Care. 2005; 28(1):27-32.

33. SCHMIDT AM, et al. Advanced glycation endproducts (AGEs) induce oxidant stress in the gingiva: a potential mechanism underlying accelerated periodontal disease associated with diabetes. J Periodontal Res. 1996; 31(7):508-515.

34. TAYLOR GW, et al. Severe periodontitis and risk for poor glycemic control in patients with non-insulin-dependent diabetes mellitus. J Periodontol. 1996; 67(10 Suppl):1085-1093.

35. TAYLOR JJ, et al. A review of the evidence for pathogenic mechanisms that may link periodontitis and diabetes. $J$ ClinPeriodontol. 2013; 40 Suppl14:S113-34. 
36. TESHOME A, YITAYEH A. The effect of periodontal therapy on glycemic control and fasting plasma glucose level in type 2 diabetic patients: systematic review and meta-analysis. BMC Oral Health. 2016; 17(1):31.

37. TSOBGNY-TSAGUE NF, et al. Effects of nonsurgical periodontal treatment on glycated haemoglobin on type 2 diabetes patients (PARODIA 1 study): a randomized controlled trial in a sub-Saharan Africa population. BMC Oral Health. 2018; 18(1):28.

38. VERGNES JN, et al. The effects of periodontal treatment on diabetic patients: The DIAPERIO randomized controlled trial. J ClinPeriodontol. 2018; 45(10):1150-1163.

39. WU Y, et al. Diabetes mellitus related bone metabolism and periodontal disease. Int J Oral Sci. 2015; 7(2):63-72.

40. YOON M, et al. Characterisation of advanced glycation endproducts in saliva from patients with diabetes mellitus. BiochemBiophys Res Commun, 2004; 323(2):377-381.

41. ZIZZI A, et al. Gingival advanced glycation end-products in diabetes mellitus-associated chronic periodontitis: an immunohistochemical study. J Periodontal Res. 2013; 48(3):293-301. 